Bundesgesundheitsbl 2017 · 60:1328-1335 https://doi.org/10.1007/s00103-017-2639-1 Online publiziert: 23. Oktober 2017 (c) Der/die Autor(en) 2017. Dieser Artikel ist eine Open-Access-Publikation.

CrossMark

\section{Tobia Lakes}

Geographisches Institut, Integrative Research Institute on Transformations of Human-Environment Systems (IRI THESys), Humboldt-Universität zu Berlin, Berlin, Deutschland

\title{
Eine räumlich differenzierte Analyse von Verkehrsunfällen mit Radfahrer- und Fußgänger- beteiligung in Berlin
}

\section{Hintergrund}

In vielen deutschen Kreisen und kreisfreien Städten wurden in den letzten Jahren Fußgänger- und Radverkehrskonzepte entwickelt, um die ehemals und insbesondere in der Nachkriegszeit auf den Autoverkehr konzentrierte Siedlungsentwicklung an aktuelle Ziele einer nachhaltigen Verkehrsentwicklung anzupassen. Von besonderer Bedeutung ist dies in wachsenden Städten wie Berlin, in denen die Verdichtung zu einer stetigen Zunahme des innerstädtischen Straßenverkehrs führt. Die gegenwärtigen Konzepte zielen insbesondere auch auf gesundheitsrelevante Aspekte ab, nämlich auf die Reduktion von Verkehrsaufkommen, und damit weniger Lärm- und Luftbelastung, und auf die Förderung von aktiver Mobilität und den damit einhergehenden Gesundheitseffekten [1, 2]. Insbesondere vor dem Hintergrund der Diskussionen zur Bewegungsfreundlichkeit von Räumen und der hohen und zum Teil steigenden Verbreitung von Übergewicht und Adipositas in Deutschland ist diese Entwicklung aus gesundheitswissenschaftlicher Sicht sehr zu begrüßen, denn die aktive Alltagsmobilität trägt einen wesentlichen Teilaspekt zur physischen Mobilität der städtischen Bevölkerung bei [3-6].

Gleichwohl stehen den vielen Vorteilen der aktiven Mobilität, insbesondere der Rad- und Fußgängermobilität, gesundheitliche Folgen im städtischen Raum gegenüber, die noch weitgehend untererforscht sind. Im Vordergrund stehen Verkehrsunfälle und die dadurch bedingten Verletzungen und Todesfälle, die insbesondere in verkehrlich intensiv genutzten Räumen eine wichtige Rolle in der Gesundheitsstatistik einnehmen. Darüber hinaus sind gesundheitliche Auswirkungen auf Atemwegserkrankungen durch Luftverschmutzung nicht zu vernachlässigen. Strategien, die die aktive Mobilität in Städten fördern sollen, müssen daher insbesondere auf die Reduktion von Verkehrsunfällen abzielen und eine sichere Infrastruktur bereitstellen [2]. Der Allgemeine Deutsche Fahrradclub (ADFC) kritisiert in Berlin z. B., dass die Unfallbrennpunkte zwar seit Jahren bekannt seien, aber dennoch in der Radverkehrsführung im Wesentlichen unverändert belassen wurden [7]. So verunglückten allein im Jahr 2016 19 Verkehrsteilnehmer bei Verkehrsunfällen mit Radfahrerbeteiligung in Berlin tödlich. Insgesamt bedeutet das, dass jeder 3. Verkehrstote bei einem Unfall mit Radfahrerbeteiligung zu beklagen war [8].

Eine wichtige Voraussetzung für die Entwicklung zukünftiger Strategien ist die räumlich und zeitlich möglichst detaillierte Analyse von Verkehrsunfällen, um im Sinne der Prävention Unfallschwerpunkte $\mathrm{zu}$ identifizieren und durch entsprechende Maßnahmen $\mathrm{zu}$ adressieren. Existierende Studien zu Verkehrsunfällen differenzieren bereits nach Teilräumen, wie z. B. administrativen Einheiten der Bezirke in Berlin [9]. Allerdings werden dabei aggregierte Daten verwendet, die das Potenzial der räumlich expliziten Analyse von durch Koordinaten verortbaren Unfallorten nicht ausschöpfen. Die Polizeistatistik erfasst umfangreiche Daten auf Punktebene, die räumlich verortet sind und somit eine integrierte räumliche und zeitliche Analyse mit geografischen Informationssystemen (GIS) ermöglichen. Solche differenzierten Datengrundlagen können mit den entsprechenden Studien einen wesentlichen Beitrag zur Gesundheitsprävention beitragen, werden in der sektoral zwischen Mobilität, Umwelt und Stadtentwicklung getrennten Gesundheitsberichterstattung in Deutschland bislang jedoch nur selten berücksichtigt [10]. Dies mag an unterschiedlichen Herangehensweisen der Analyse und Themen liegen. Insbesondere bei der Analyse von Verkehrsunfällen ist eine interdisziplinäre Herangehensweise von Gesundheits-, Verkehrs- und Raumwissenschaftlern notwendig [11]. Nur so können koordinierte Stadtentwicklungsmaßnahmen und gesundheitspolitische Maßnahmen zur Förderung aktiver Mobilität und zum Abbau von Mobilitätsbarrieren in städtischen Räumen entwickelt werden.

Studien $\mathrm{zu}$ Unfalluntersuchungen mit Radfahrer- und Fußgängerbeteiligung zeigen, dass es eine Vielzahl an Determinanten für das Auftreten von Verkehrsunfällen gibt [12-14]. So können bestimmte verkehrliche, betriebliche oder entwurfstechnische Faktoren unfallbegünstigend oder auch unfallvermeidend wirken. Oft tragen auch Mängel in der Ausgestaltung von Knotenpunkten 


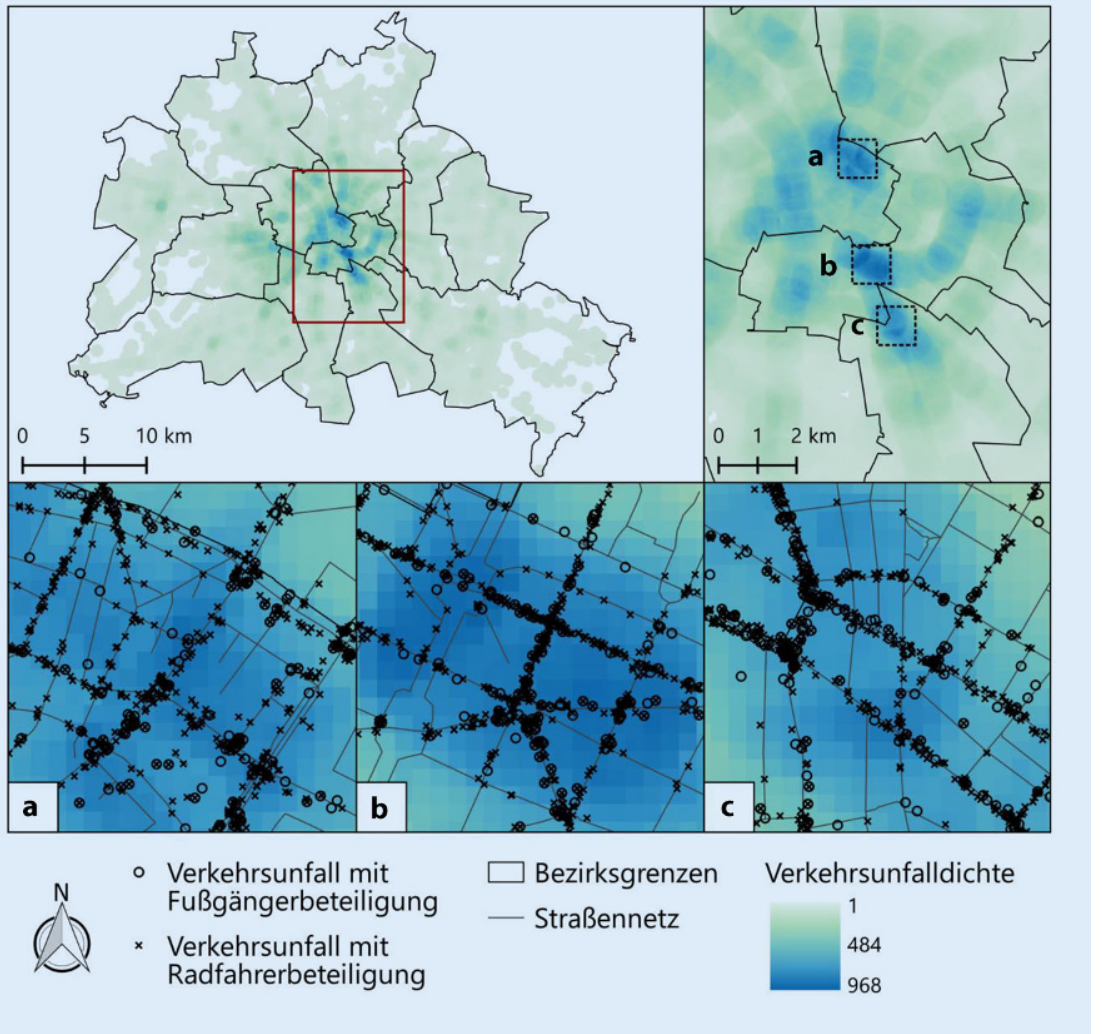

Abb. 1 \& Punktdichtekarte der Verkehrsunfälle mit Fußgänger- und Radfahrerbeteiligung (20112015) mit Detailansichten für die Hotspots um den Alexanderplatz (a), Moritzplatz (b) und Hermannplatz (c)

zum Unfallgeschehen bei. So stellen sich gerade bei den Abbiegeunfällen mit Radfahrer- oder Fußgängerbeteiligung besonders oft Beeinträchtigungen der Sicht zwischen den Verkehrsteilnehmenden als problematisch heraus [15]. Weitere Faktoren, wie z.B. die Schuldfrage der Unfallbeteiligten, Rotmissachtung, Alkoholeinfluss, überhöhte Geschwindigkeit oder linksfahrende Radfahrer, spielen eine ebenso wichtige Rolle.

Kleinräumige und räumlich und zeitlich differenzierte Analysen der polizeilichen Unfallstatistik mithilfe von GIS bieten ergänzende Möglichkeiten der Exploration und damit auch Identifikation von möglichen Unfallschwerpunkten und -ursachen sowie die Ableitung von Handlungsempfehlungen. Basierend auf räumlich-zeitlichen Musteranalysen der Verkehrsunfallvorkommen können Hypothesen über zugrunde liegende Prozesse und Korrelationen formuliert und getestet werden. Eine erste komparative Studie auf der Ebene von Gemeinden schlussfolgert beispielsweise, dass die und unangemessene zu vermeiden [15, 19].

Ziele der vorliegenden Studie sind demzufolge anhand des Fallbeispiels Berlins:

1. die Identifikation von räumlichen und zeitlichen Mustern der Verteilung von Unfällen mit Radfahrerund Fußgängerbeteiligung,

2. die Identifikation von Hotspots und Exploration möglicher Ursachen und

3. die kritische Diskussion von Möglichkeiten und Grenzen der Ergebnisse und die Ableitung von Handlungsempfehlungen.

\section{Daten und Methoden}

\section{Untersuchungsgebiet}

Als Untersuchungsgebiet wurde die Bundeshauptstadt Berlin gewählt, die in den letzten Jahren stetig an Einwohnerzahlen gewonnen hat. Der Anteil des Radfahrverkehrs am Gesamtverkehrsaufkommen hat in den letzten Jahren kontinuierlich zugenommen, auch wenn repräsentative Daten hierzu fehlen [1, $7,8]$. Gegenwärtig wird eine neue Radverkehrsstrategie und -politik intensiv diskutiert. Zu Fuß zurückgelegte Wegstrecken entsprechen zeitlich etwa denen der mit dem Auto zurückgelegten Wege in Berlin [1]. Aufgrund der heterogenen Nutzung und Bebauung sind die Einwohnerzahlen und das Verkehrsaufkommen sehr unterschiedlich verteilt. Es kann von einem deutlich höheren Verkehrsaufkommen im Innenstadtbereich im Vergleich zum Außenbereich ausgegangen werden.

\section{Daten} teiligung identifizieren [17]. Loidl et al. [18] fassen für ihre Fallstudie aus Österreich einige wesentliche methodische Anforderungen an die Auswertung von Verkehrsunfällen mit Radfahrerbeteiligung zusammen und zeigen Potenziale der räumlich-zeitlichen Datenexploration auf. Solche Studien können eine Grundlage für die verstärkt geforderten evidenzbasierten Ansätze zur Sicherheit bei der aktiven Mobilität darstellen, um wirksame Maßnahmen zu identifizieren
Für diese Studie wurden die Verkehrsunfalldaten seitens des Polizeipräsidenten Berlins zur Verfügung gestellt. Die Daten umfassen den Zeitraum vom 01.01.2011 bis zum 31.12.2015. Es sind Unfälle in Form von geocodierten Punkten mit anonymisierten Informationen über den jeweiligen Unfall aufgeführt. Die Daten stehen in Tabellenform zur Verfügung, aufgegliedert in einen Datensatz mit Verkehrsunfällen mit Fußgängerbeteiligung sowie einen Datensatz mit Unfällen mit 
Bundesgesundheitsbl 2017·60:1328-1335 https://doi.org/10.1007/s00103-017-2639-1

(c) Der/die Autor(en) 2017. Dieser Artikel ist eine Open-Access-Publikation.

\section{T. Lakes}

\section{Eine räumlich differenzierte Analyse von Verkehrsunfällen mit Radfahrer- und Fußgängerbeteiligung in Berlin}

\section{Zusammenfassung}

Hintergrund. Verkehrskonzepte, die den Fußgänger- und Radfahrerverkehr fördern sollen, werden derzeit in vielen deutschen Kreisen und kreisfreien Städten entwickelt. Eine kleinräumige Analyse des Auftretens und möglicher Ursachen von Verkehrsunfällen ist dabei aus stadtplanerischer, verkehrspolitischer und gesundheitswissenschaftlicher Sicht entscheidend.

Ziele. 1) Die Identifikation von räumlichen und zeitlichen Mustern der Verteilung von Unfällen mit Radfahrer- und Fußgängerbeteiligung, 2) die Identifikation von Hotspots und Exploration möglicher Ursachen und 3) die kritische Diskussion der Ergebnisse und die Ableitung von übertragbaren Handlungsempfehlungen.
Material und Methoden. Es werden räumlich-zeitliche Verteilungen von Daten der Unfallstatistik Berlin mit Fußgängerund Radfahrerbeteiligung von 2011 bis 2015 unter Anwendung von geografischen Informationssystemen (GIS) untersucht. Ergebnisse. Die Gesamtzahl der Unfälle mit Fußgänger- und Radfahrerbeteiligung in Berlin bleibt jeweils relativ stabil. Es treten signifikante Häufungen (räumliche Hotspots) auf mit einer starken Konzentration im Innenstadtbereich.

Schlussfolgerungen. In einer kritischen Diskussion werden der Mehrwert von geografischen Konzepten identifiziert, z. B. die räumlich explizite Verortung von gesundheitsrelevanten Daten (hier:
Verkehrsunfalldaten), die Bedeutung der Integration von anderen Datenquellen zur Auswertung der gesundheitlichen Belastung von Räumen (hier: Verkehrsunfallstatistik der Polizei) und die Möglichkeiten und Grenzen der räumlich-zeitlichen Datenauswertung (hier: räumliche Punktdichteanalysen) für die Ableitung von entscheidungsunterstützenden Empfehlungen und zur Evaluation von Maßnahmen der Gesundheitsprävention und der gesundheitsrelevanten Stadtentwicklung.

Schlüsselwörter

Verkehrsunfälle $\cdot$ Kleinräumige Variation · Radverkehr · Fußgängerverkehr . Stadtentwicklung

\section{A spatially explicit analysis of traffic accidents involving pedestrians and cyclists in Berlin}

\section{Abstract}

Background. In many German cities and counties, sustainable mobility concepts that strengthen pedestrian and cyclist traffic are promoted. From the perspectives of urban development, traffic planning and public healthcare, a spatially differentiated analysis of traffic accident data is decisive.

Objectives. 1) The identification of spatial and temporal patterns of the distribution of accidents involving cyclists and pedestrians, 2) the identification of hotspots and exploration of possible underlying causes and 3 ) the critical discussion of benefits and challenges of the results and the derivation of conclusions.
Material and methods. Spatio-temporal distributions of data from accident statistics in Berlin involving pedestrians and cyclists from 2011 to 2015 were analysed with geographic information systems (GIS).

Results. While the total number of accidents remains relatively stable for pedestrian and cyclist accidents, the spatial distribution analysis shows, however, that there are significant spatial clusters (hotspots) of traffic accidents with a strong concentration in the inner city area.

Conclusions. In a critical discussion, the benefits of geographic concepts are identified, such as spatially explicit health data (in this case traffic accident data), the importance of the integration of other data sources for the evaluation of the health impact of areas (traffic accident statistics of the police), and the possibilities and limitations of spatialtemporal data analysis (spatial point-density analyses) for the derivation of decisionsupported recommendations and for the evaluation of policy measures of health prevention and of health-relevant urban development.

Keywords

Traffic accidents $\cdot$ Spatial variations $\cdot$ Bike traffic · Pedestrians · Urban development
Radfahrerbeteiligung. Auch wenn die Daten von hoher zeitlicher, räumlicher und thematischer Genauigkeit sind, so muss bei der folgenden Auswertung dennoch von einer Unterschätzung der Unfallhäufigkeiten ausgegangen werden. Es ist sehr wahrscheinlich, dass viele Unfälle, insbesondere ohne körperliche Verletzungen oder Sachbeschädigung, sowie auch Alleinunfälle ohne Beteiligung weiterer Verkehrsteilnehmer, nicht bei der Polizei gemeldet werden.

\section{Methoden}

Zur Analyse und Weiterverarbeitung der zugrunde liegenden Vektordaten wurde ArcGIS 10.4 von ESRI Inc. (Redlands, CA, USA; [20]) verwendet. Zunächst wurden die geocodierten Daten eingelesen und für Unfälle mit Radfahrer- bzw. Fußgängerbeteiligung räumlich visualisiert. Im zweiten Schritt wurden Dichtekarten erstellt, um gezielt Hotspots im Stadtraum identifizieren zu können, die eine Häufung von Unfällen aufweisen. Bei der Punktdichteanalyse wird die An- zahl der Unfälle in einem definierten Radius um den Mittelpunkt jeder Zelle ermittelt und anschließend durch die Fläche dieses Umkreises dividiert und der Rasterzelle zugeordnet. Für diese Studie wurde eine Zellgröße von $50 \mathrm{~m}$ und ein Radius von $500 \mathrm{~m}$ verwendet, um eine räumlich möglichst hoch aufgelöste Information zu erhalten. Zudem kann davon ausgegangen werden, dass sich mit einer Distanz von mehr als $500 \mathrm{~m}$ eine neue verkehrliche Situation ergibt. Um Aussagen über die zeitliche Entwicklung von Verkehrsunfällen und den damit ver- 


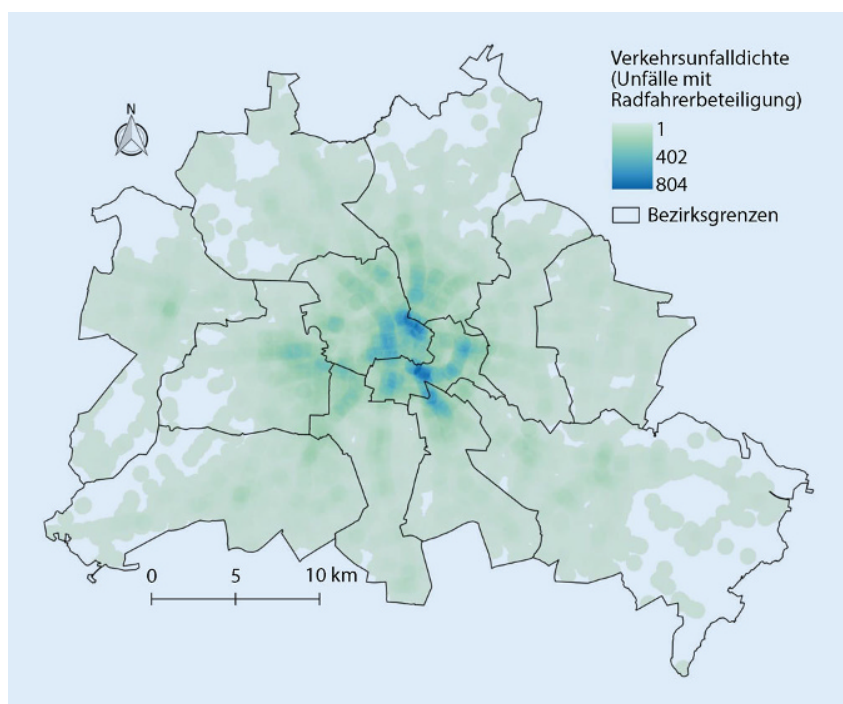

Abb. $2 \Delta$ Punktdichtekarte der Verkehrsunfälle mit Radfahrerbeteiligung (2011-2015)

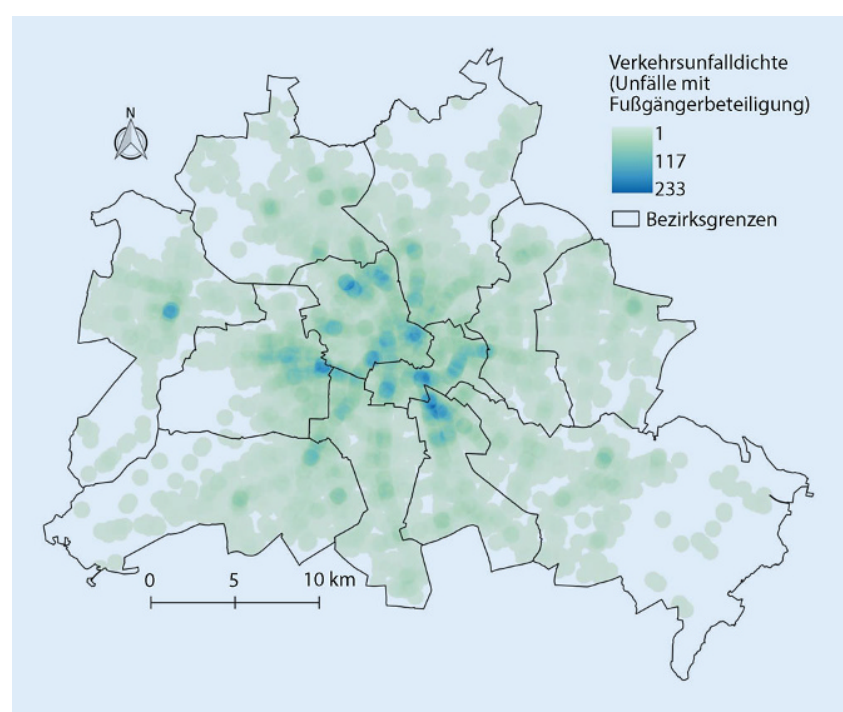

Abb. 3 ॥ Punktdichtekarte der Verkehrsunfälle mit Fußgängerbeteiligung (2011-2015) bundenen potenziellen Hotspots treffen zu können, wurden Dichtekarten separat für jedes Jahr angefertigt, sowohl für Verkehrsunfälle mit Radfahrer- als auch mit Fußgängerbeteiligung.

\section{Ergebnisse}

Räumlich-zeitliche Verteilung der Verkehrsunfälle mit Fußgängerund Radfahrerbeteiligung

Insgesamt umfasst der Datensatz im Zeitraum 2011-2015 37.094 Unfälle mit Radfahrer- sowie 12.537 Unfälle mit Fußgängerbeteiligung im Berliner Stadtgebiet. Da Fußgänger und Radfahrer in einen Verkehrsunfall verwickelt sein können, kommt es zu Überschneidungen in den Datensätzen, sodass 2227 Verkehrsunfälle mit Beteiligung von beiden Gruppen angegeben werden.

Die räumliche Verteilung der Verkehrsunfälle zeigt sich in der kartografischen Darstellung der geocodierten Verkehrsunfallpunkte in $\bullet$ Abb. 1. Ebenfalls dargestellt wird die Dichte eines Verkehrsunfalls im Untersuchungszeitraum mit bis zu 968 Verkehrsunfällen. Für den Stadtraum in Berlin kann für den Untersuchungszeitraum festgestellt werden, dass eine Konzentration im Innenstadtbereich sowohl für die Verkehrsunfälle mit Fußgänger- als auch mit Radfahrerbeteiligung gegeben ist, während in den Außenbereichen deutlich niedrigere Zahlen registriert werden (vgl. • Abb. 1).

Eine differenzierte Betrachtung von Verkehrsunfällen nach Fußgänger- und Radfahrerbeteiligung zeigt ebenfalls die Konzentration im Innenstadtbereich, weist aber auch auf spezifische räumliche Konzentrationen hin (vgl. - Abb. 2 und 3). So können für Verkehrsunfälle mit Radfahrerbeteiligung einige spezifische Hotspots im Innenstadtbereich identifiziert werden, die relativ hohe Dichten (bis zu 804) über den Untersuchungszeitraum aufweisen (vgl. - Abb. 2). Bei den Fußgängern ist die Verteilung räumlich differenzierter mit insgesamt weniger hohen Dichten (bis $\mathrm{zu}$ 233) und verschiedenen Hotspots verteilt im Stadtgebiet.

Die Darstellung der jährlichen Entwicklung und räumlichen Verteilung der Dichten der Verkehrsunfälle mit Fußgänger- und Radfahrerbeteiligung zeigt das gleiche Muster. Auch wenn kleinere Unterschiede identifiziert werden können, so scheint es sich jedoch weitestgehend um die gleichen Unfallhotspots zu handeln (vgl. 0 Abb. 4 und 5).

Die zeitliche Verteilung der Verkehrsunfälle mit Radfahrerbeteiligung (vgl. - Abb. 6) zeigt einen deutlichen saisonalen Verlauf: In den Frühjahrs- und Sommermonaten wird das Fahrrad häufiger genutzt und es kommt vermehrt zu
Verkehrsunfällen. Die niedrigsten Werte sind Ende Dezember und Anfang Januar sowie in der Urlaubszeit im Sommer in allen Jahren zu verzeichnen.

\section{Mögliche Ursachen der Unfallhäufungen}

Differenziert man die räumlichen Verteilungen nach verschiedenen Unfallarten, so sind noch deutlichere räumliche Konzentrationen als bei den Verteilungen aller Verkehrsunfälle identifizierbar. Dies trifft insbesondere auf die Verkehrsunfälle mit Radfahrerbeteiligung zu. Hier können, basierend auf bisherigen Studien, unfallbegünstigende oder unfallvermeidende Determinanten herangezogen werden. Dabei sind insbesondere Faktoren wie die Vorfahrtsregelung (z. B. Beschilderung, „rechts vor links"), Sichtbedingungen, farbliche Markierungen oder die Frage nach Radweg oder Fahrradstreifen auf der Fahrbahn entscheidend. Eine detaillierte Analyse der Verkehrsführung der einzelnen Verkehrsunfallhotspots ermöglicht dann im nächsten Schritt, nachteilige oder fehlerhafte Verkehrslenkungen zu identifizieren und Verbesserungsmöglichkeiten aufzuzeigen (vgl. @ Abb. 1).

So hat die Analyse gezeigt, dass einige Unfallursachen gehäuft vorkommen, wie beispielsweise die Rechtsabbiegerproblematik, d.h. PKW-Rechtsabbieger 

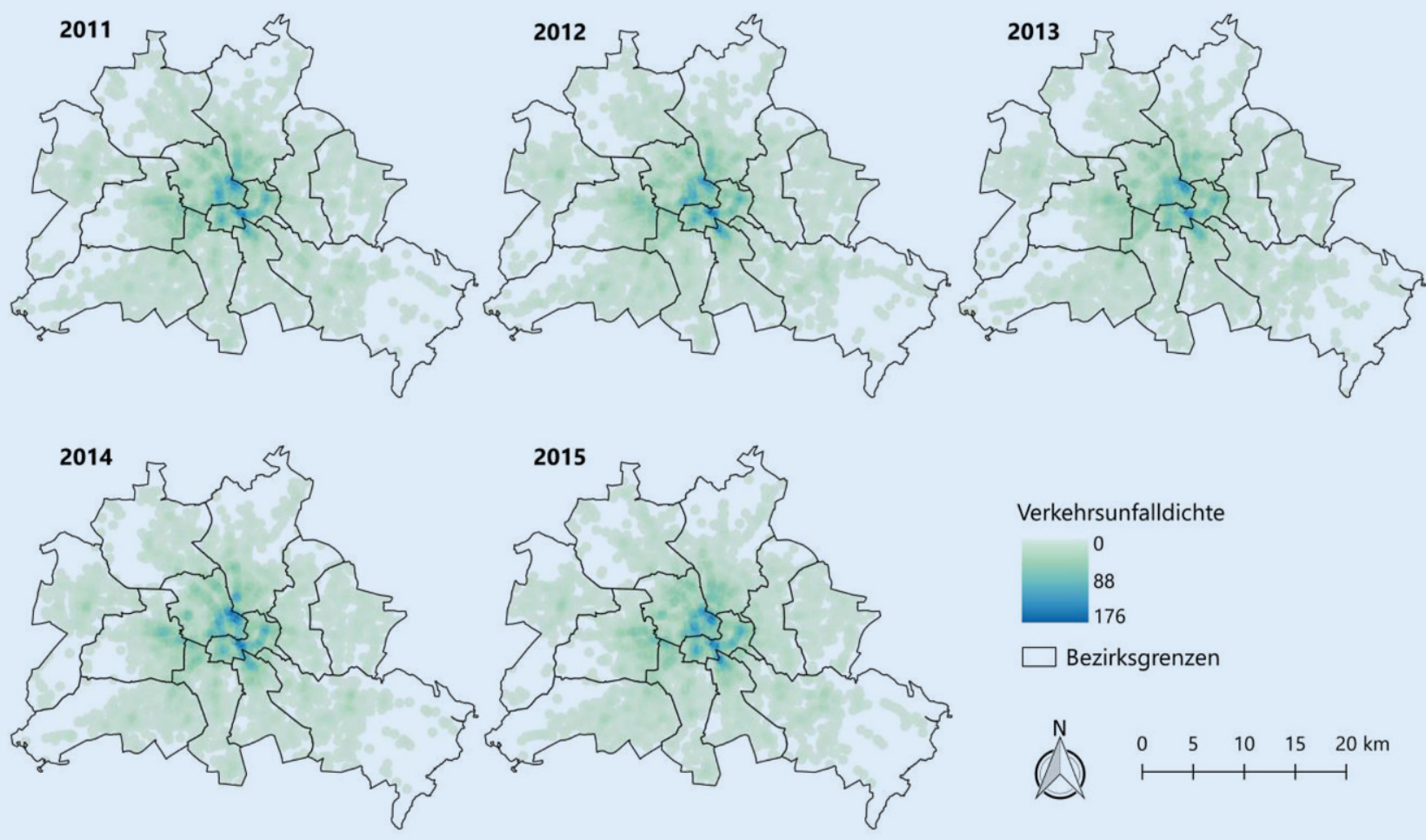

Abb. 4 A Entwicklung der Verteilung der Verkehrsunfälle mit Radfahrerbeteiligung (2011-2015)

übersehen den geradeausfahrenden Radfahrerverkehr. Ein Hotspot ist hier der Moritzplatz, ein Kreisverkehr mit Fahrradstreifen und ohne Lichtsignalanlagen (d. h. Ampeln). Dort tritt häufig als eine Verkehrsunfallursache die Missachtung der Vorfahrt der Radfahrer seitens der PKW-Fahrer auf. Neben baulichen Maßnahmen, die diese Probleme adressieren können, muss aber auch immer berücksichtigt werden, dass viele Unfälle von den Radfahrern mitverschuldet sind, die sowohl intentional als auch aus Unwissenheit geschehen können.

\section{Diskussion und \\ Schlussfolgerungen}

Im Rahmen dieser Studie wurden kleinräumige Differenzierungen in Verkehrsunfällen in Berlin mit Radfahrer- und Fußgängerbeteiligung mithilfe von GIS untersucht. Ziele der vorliegenden Studie waren (1) die Identifikation von räumlichen und zeitlichen Mustern der Verteilung von Unfällen mit Radfahrerund Fußgängerbeteiligung, (2) die Identifikation von Hotspots und Exploration möglicher Ursachen und (3) die kritische Diskussion von Möglichkeiten und Grenzen der Ergebnisse sowie die Ableitung von Handlungsempfehlungen. Wie in den Abbildungen und Karten gezeigt werden konnte, zeigte sich eine räumliche Häufung von Verkehrsunfällen an spezifischen Orten, die teilweise über Jahre hinweg bestehen. Auch bei der zeitlichen Trendanalyse konnten innerhalb der Jahre spezifische Zeiträume mit Häufungen von Verkehrsunfällen identifiziert werden.

Gleichwohl müssen die Ergebnisse dieser Studie vor dem Hintergrund einiger Grenzen kritisch gesehen werden. So sind keine räumlich flächendeckenden Daten über die Zahl und die Charakteristika (z. B. Geschlecht, Alter) der Radfahrer und Fußgänger für Berlin verfügbar [21]. Auch andere Studien [16, 18] stehen vor diesem Problem, denn eine Auswertung nach möglichen Ursachen, Risikoabschätzungen und insbesondere auch die Identifikation von Zielgruppen, die besonders erreicht werden sollten, ist damit nicht möglich. Darüber hinaus bedarf es der spezifischen Berücksich- tigung von räumlichen Phänomenen in den Daten, wie beispielsweise der räumlichen Konzentration von Verkehrsunfällen an Straßen-/Wegeverläufen. Das bedeutet, dass viele weiterführende Methoden des räumlich-zeitlichen Scans oder der Punktdichteanalyse ungeeignet sind und kritisch reflektiert werden müssen [18]. Dies konnte im Rahmen dieser ersten einführenden Studie nicht im Detail herausgearbeitet werden, da die dafür notwendigen detaillierten $\mathrm{Da}$ ten über Straßen- und Wegenetze nicht zur Verfügung standen.

Im Folgenden werden nun zunächst die Möglichkeiten und Grenzen der kleinräumigen Analyse von Verkehrsunfällen mit GIS diskutiert, wobei vier entscheidende Aspekte angeführt werden: die geometrischen und topologischen Dimensionen von Daten, die räumliche und zeitliche Exploration von Mustern, die Integration von verschiedenen Datenquellen und die spezifischen Analysemethoden. Die geometrischen und topologischen Dimensionen von Daten und Phänomenen - hier am Beispiel der exakten Positionierung und 

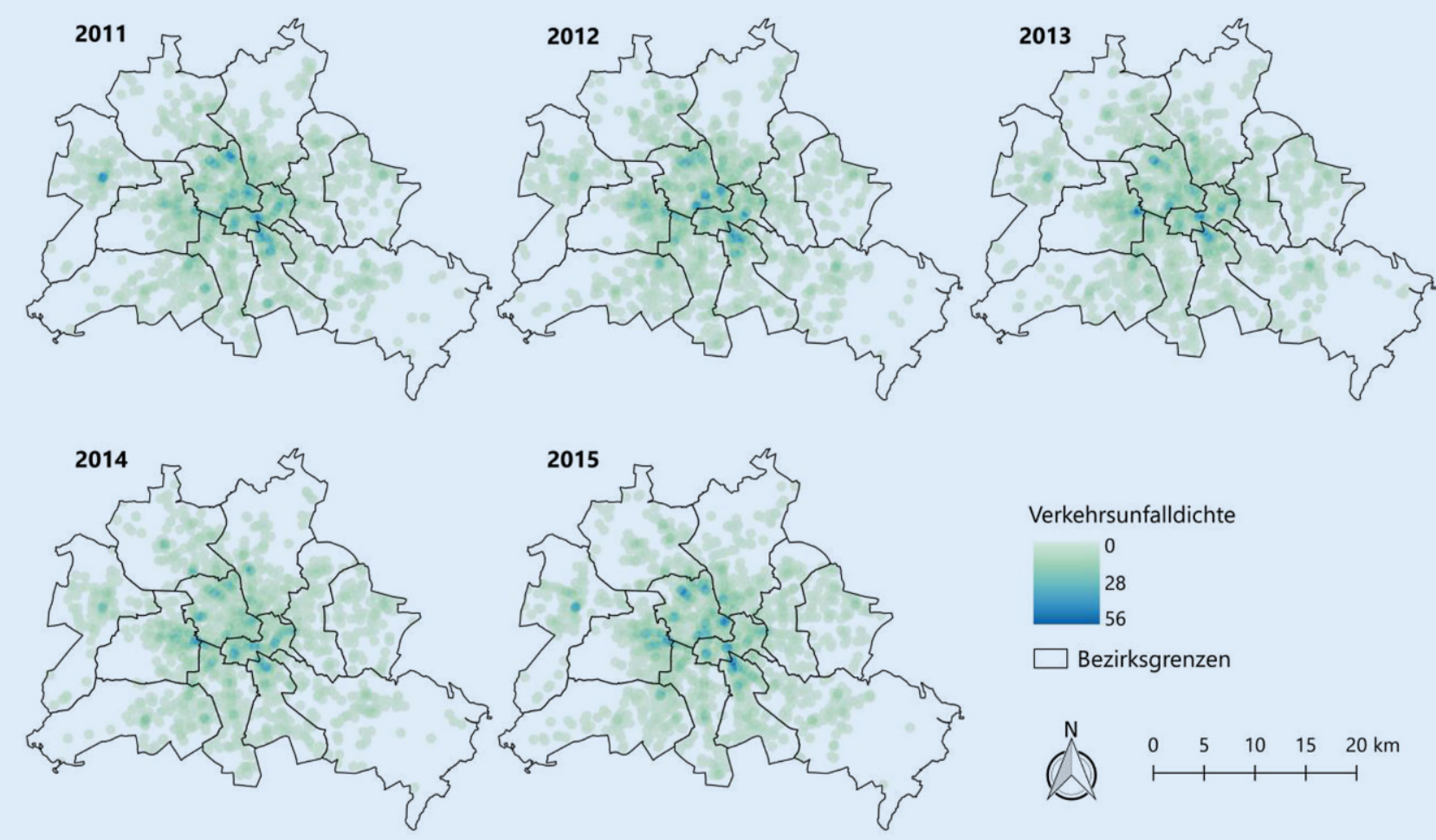

Abb. 5 Entwicklung der Verteilung der Verkehrsunfälle mit Fußgängerbeteiligung (2011-2015)

Verortung (Geometrie) von Verkehrsunfällen - ermöglicht es, weitere Aussagen über den Raum und die Ursachen abzuleiten und Unfallschwerpunkte zu identifizieren. Die topologische Dimension ermöglicht dabei die Betrachtung von Nachbarschaftsbeziehungen zwischen den Objekten selbst (also z. B. die räumliche Clusterung von Unfällen) oder aber auch zwischen verschiedenen Objekten (z. B. der Zusammenhang zwischen Unfällen und einer bestimmten baulichen Ausgangssituation). Die so in Raum und Zeit verorteten Daten bieten die Grundlage für räumlich-zeitliche $\mathrm{Da}$ tenexplorationen, um von identifizierten Mustern auf die zugrunde liegenden Ursachen zu schließen bzw. Hypothesen zu formulieren. Weiterführende Analysen mit entsprechenden Hintergrundinformationen können z. B. auf erklärende Ansätze abzielen und differenzierte Ursachenforschung oder Risikoforschung betreiben und weitere Datensätze hinzufügen $[16,17,22]$. Untersuchungen zur Walkability (Fußgängerfreundlichkeit) und Bikeability (Fahrradfreundlichkeit) können solche Unfallanalysen ergänzen
$[4,23]$. Dabei erlauben GIS die flächendeckende Abschätzung der Fußgängerbzw. Radfahrerfreundlichkeit von Räumen über definierte fördernde oder hemmende Kriterien [24, 25]. Solche Ansätze können einer ersten Abschätzung dienen und damit auch die Räume identifizieren, in denen politische oder planerische Maßnahmen zur Förderung oder Verbesserung/Sicherung der aktiven Mobilität notwendig sind.

Darüber hinaus ist die explizite Berücksichtigung und Definition von geeigneten räumlichen und zeitlichen Skalen und Aggregationsniveaus entscheidend. In dieser Studie wurde z. B. eine räumlich explizite Darstellung auf Punktebene verwendet und die Anzahl der Punkte in einem definierten Radius pro Zelle (Dichtekarte) visualisiert. Zeitlich wurde eine Aggregation auf Wochen- bzw. Jahresebene gewählt; eine detailliertere Analyse der Daten auf Tages-bzw. Stundenebene wäre zukünftig ebenfalls möglich. Es gilt dabei zu berücksichtigen, dass eine räumliche Aggregation immer mit einem Informationsverlust einhergeht, der sich in der Verringerung der lokalen Variabi- lität abbildet. Mit dem „modifiable areal unit problem“ (MAUP) wird dies explizit adressiert: $\mathrm{Ob}$ administrative Grenzen oder Pixel - die Grenzen dieser Untersuchungseinheiten sind veränderbar und häufig nicht studienspezifisch definiert [18].

Die Integration von unterschiedlichen Datenquellen zur Analyse von gesundheitswissenschaftlichen Phänomenen ist von großer Bedeutung. GIS ermöglichen dabei die Integration verschiedener Datenquellen und -typen über den Raumbezug, sodass der Mehrwert von geocodierten Daten und Koordinatenform deutlich wird [10]. Entscheidend wird in Zukunft die Dokumentation und Verfügbarmachung von verschiedenen Datenquellen sein. Best-PracticeBeispiele zur Integration können hier den Weg für operationelle und vergleichbare Anwendungen ebnen. AuBerdem sind die Analysemethoden an die entsprechenden Herausforderungen der Datengrundlage und Fragestellung anzupassen. So ergeben sich Besonderheiten von Punktmusteranalysen im Vergleich zu aggregierten Datenanaly- 

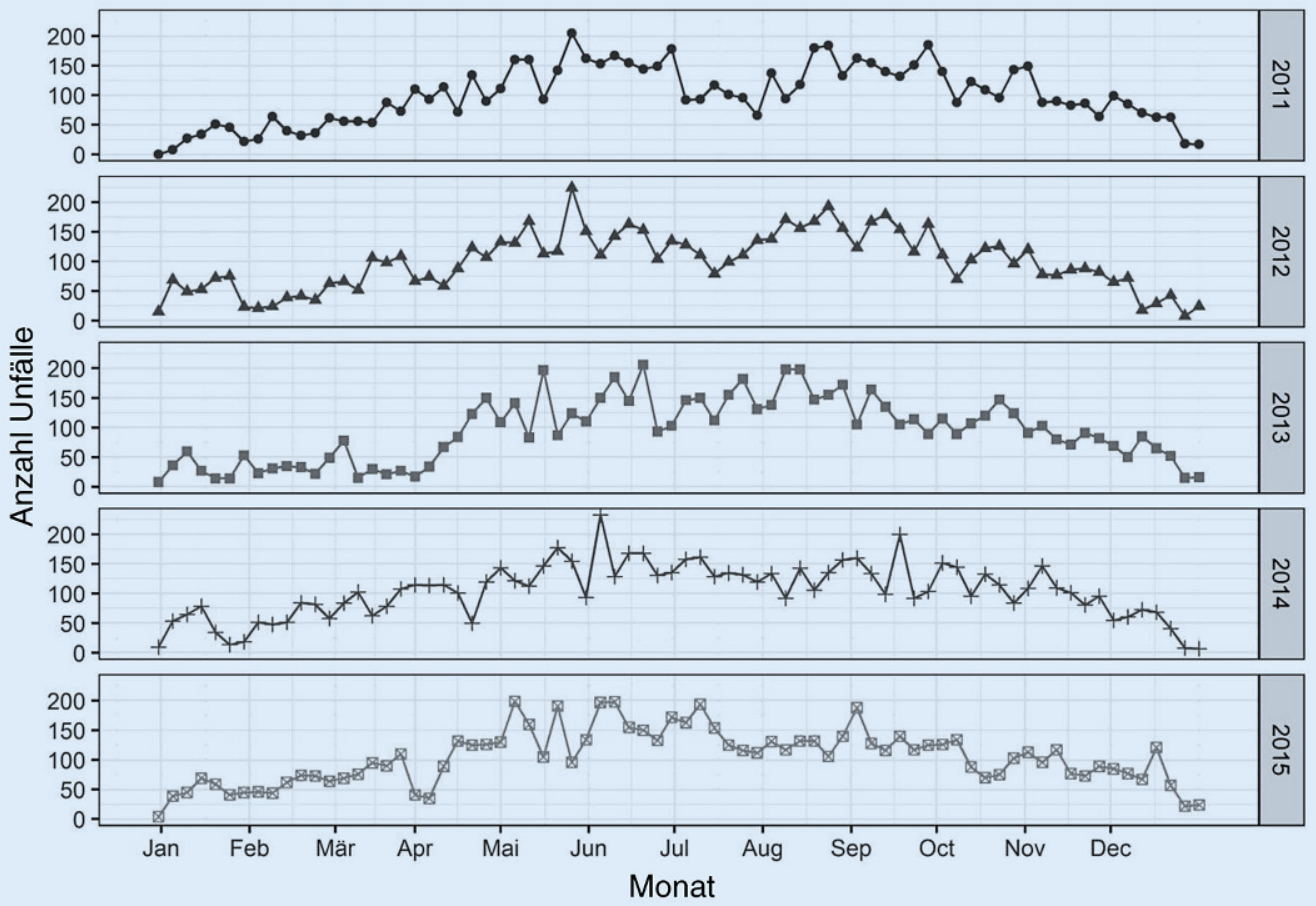

$\rightarrow 2011 \_2012-2013+2014 \div 2015$
Abb. $6 \triangleleft$ Zeitliche Verteilung der Verkehrsunfälle mit Radfahrerbeteiligung im Jahresverlauf (2011-2015) sen, denn das Sampling muss ebenso berücksichtigt werden wie die Tatsache, dass keine Daten über das Aufkommen von Radfahrern/Fußgängern bzw. über Anteile bestimmter Bevölkerungsgruppen verfügbar sind.

Zusammengefasst zeigen die Ergebnisse dieser Datenanalyse folgende methodische wie handlungsrelevante Erkenntnisse auf: (1) den Mehrwert der Analyse von räumlich explizit über Punktdaten verortbaren Gesundheitsdaten (hier: Verkehrsunfalldaten), (2) die Bedeutung der Integration von anderen Datenquellen zur Auswertung der gesundheitlichen Belastung von Räumen (hier: Verkehrsunfallstatistik der Polizei) und (3) die Möglichkeiten und Grenzen der räumlich-zeitlichen Datenauswertung (hier: räumliche Punktdichteanalysen) für die Ableitung von entscheidungsunterstützenden Empfehlungen und zur Evaluation von politischen Maßnahmen der Gesundheitsprävention und auch der gesundheitsrelevanten Stadtentwicklung. Zukünftig werden in Zeiten von „big geodata“ explorative Datenanalysen und Data-Mining verstärkt an Bedeutung gewinnen und bieten neue Möglichkeiten der Analyse. Weiterführender Forschungsbedarf ergibt sich insbesondere im Hinblick auf eine detaillierte Analyse der Unfallursachen sowie auf eine zielgruppenspezifische Auswertung der Daten, wie beispielsweise von Kindern oder älteren Bevölkerungsgruppen.

\section{Korrespondenzadresse}

\section{Prof. Dr. T. Lakes}

Geographisches Institut, Integrative Research Institute on Transformations of Human-

Environment Systems (IRI THESys), HumboldtUniversität zu Berlin

Unter den Linden 6, 10099 Berlin, Deutschland Tobia.Lakes@hu-berlin.de

Danksagung. Die Autorin dankt dem Polizeipräsidenten von Berlin für die Bereitstellung der Daten. Den Studierenden des Geographischen Instituts der Humboldt-Universität sei für ihre Teilbeiträge zur Aufbereitung und Analyse der Daten gedankt. Besonderer Dank gilt Fabian Thiel und Dr. Florian Gollnow.

\section{Einhaltung ethischer Richtlinien}

Interessenkonflikt. T. Lakes gibt an, dass kein Interessenkonflikt besteht.

Dieser Beitrag beinhaltet keine von der Autorin durchgeführten Studien an Menschen oder Tieren.

Open Access. Dieser Artikel wird unter der Creative Commons Namensnennung 4.0 International Lizenz (http://creativecommons.org/licenses/by/4.0/deed. de) veröffentlicht, welche die Nutzung, Vervielfältigung, Bearbeitung, Verbreitung und Wiedergabe in jeglichem Medium und Format erlaubt, sofern Sie den/die ursprünglichen Autor(en) und die Quelle ordnungsgemäß nennen, einen Linkzur Creative Commons Lizenz beifügen und angeben, ob Änderungen vorgenommen wurden.

\section{Literatur}

1. Senatsverwaltung für Umwelt, Verkehr und Klimaschutz (2017) Stadtentwicklungsplan Verkehr. http://www.stadtentwicklung.berlin.de/verkehr/ politik_planung/step_verkehr/index.shtml. Zugegriffen: 13 . Sept. 2017

2. Faskunger J (2013) Promoting active living in healthy cities of Europe. J Urban Health 90:142-153

3. Frank L, Sallis JF, Saelens BE et al (2010) The development of a walkability index: application to the Neighborhood Quality of Life study. Br J Sports Med 44:924-933

4. Fraser SDS, Lock K (2010) Cycling for transport and public health: a systematic review of the effect of 
the environment on cycling. Eur J Public Health 21:738-743

5. Götschi T, Garrard J, Giles-Corti B (2016) Cycling as a part of daily life: a review of health perspectives. Transp Rev 36:45-71

6. Veisten K, Flugel S, Ramjerdi F, Minken H (2011) Cycling and walking for transport: estimating net health effects from comparison of different transport model users' self-reported physical activity. Health Econ Rev 1(1):3

7. Allgemeiner Deutscher Fahrrad-Club Berlin e.V. (2016) Fahrradunfälle in Berlin. Unfallstatistik. Die wichtigsten Fakten aus der polizeilichen Unfallstatistik. http://adfc-berlin.de/radverkehr/ sicherheit/information-und-analyse/121fahrradunfaelle-in-berlin-unfallstatistik/154die-wichtigsten-fakten-aus-der-polizeilichenunfallstatistik.html.Zugegriffen: 13. Sept. 2017

8. Der Polizeipräsident von Berlin (2017) Sonderuntersuchung Radverkehrsunfälle in Berlin. https://www.berlin.de/polizei/aufgaben/ verkehrssicherheit/verkehrsunfallstatistik/\# radfahrer.Zugegriffen: 13. Sept. 2017

9. Amt für Statistik Berlin-Brandenburg (2017) Statistischer Bericht. Verkehrsunfälle in Berlin. März 2017. https://www.statistik-berlin-brandenburg. de/publikationen/stat_berichte/2017/SB_H0101-00_2017m03_BE.pdf. Zugegriffen: 13. Sept. 2017

10. Scheiner J, Holz-Rau C (2011) A residential location approach to traffic safety: two case studies from Germany. Accid Anal Prev 43:307-322

11. Loidl M, Wendel R, Zagel B (2015) Spatial analysis and modelling of bicycle accidents and safety threats. International Cycling Saftey Conference 2015, Hanover, 15-16.09.2015. Germany

12. Alrutz D, Bohle W, Müller $H$, Prahlow $H$, Hacke U, Lohmann G (2009) Unfallrisiko und Regelakzeptanz von Fahrradfahrern. Berichte der Bundesanstalt für Straßenwesen. Verkehrstechnik Heft V 184. http://bast.opus.hbz-nrw.de/ volltexte/2011/253/pdf/V184.pdf. Zugegriffen: 13. Sept. 2017

13. Utsch A (2008) Unfallrisiko von Fahrradfahrern in Berlin von 1993-2004 vor und nach der Novellierung der Straßenverkehrsordnung für Radfahrer. Freie Universität: Institut für Rechtsmedizin - Medizinische Fakultät Charité-Universitätsmedizin. Doktorarbeit.

14. Kolrep H, Leitner R, Platho C et al (2013) Abbiegeunfälle Pkw/Lkw und Fahrrad. In: Gesamtverband der Deutschen Versicherungswirtschaft (Hrsg) In . Forschungsbericht GdV,Bd. 21.

15. Zwipp H, Barthel P, Bönninger J et al (2015) Prävention von Fahrradfahrerunfällen. Z Orthop Unfall 153:177-186

16. Delmelle EC, Thill J-C, Ha H-H (2012) Spatial epidemiologic analysis of relative collision risk factors among urban bicyclists and pedestrians. Transportation (Amst) 39:433-448

17. Vandenbulcke G, Thomas I, Int Panis L (2014) Predicting cycling accident risk in Brussels: a spatial case-control approach. Accid Anal Prev 62:341-357

18. Loidl M, Wallentin G, Wendel R, Zagel B (2016) Mapping bicycle crash risk patterns on the local scale. Safety 2:17

19. Nordback K, Sellinger M (2014) Methods for estimating bicycling and walking in Washington State. WSDOT Research Report. https://www. wsdot.wa.gov/research/reports/fullreports/828. 1.pdf.Zugegriffen: 13. Sept. 2017

20. ESRI (2017) About ArcGIS. http://www.esri.com/ arcgis/about-arcgis.Zugegriffen: 13.Sept. 2017
21. Stadtplan (2016) Fahrradverkehr - Pegelzählungen Berlin. Jahresbericht 2015. http:// www.berlin.de/senuvk/verkehr/lenkung/vlb/ download/bericht_radverkehr_2015.pdf. Zugegriffen:3. Sept. 2017

22. Truong L, Somenahalli S (2011) Using GIS to identify pedestrian-vehicle crash hot spots and unsafe bus stops. JPublic Trans 14:99-114

23. Bucksch J, Schneider S (2014) Walkability - Einführung und Überblick. In: Bucksch J, Schneider $S$ (Hrsg) Walkability: Das Handbuch zur Bewegungsförderung in der Kommune. Huber, Bern, S15-26

24. Leslie E, Coffee N, FrankL, Owen N, Bauman A, Hugo G (2007) Walkability of local communities: using geographic information systems to objectively assess relevant environmental attributes. Health Place 13:111-122

25. Winters M, Brauer B, Setton EM, Teschke K (2013) Mapping bikeability: a spatial tool to support sustainable travel. Environment and planning B. Environ Plann B Urban Anal City Sci 40:865-883 